\title{
To Tune or Not to Tune? Adapting Pretrained Representations to Diverse Tasks
}

\author{
Matthew E. Peters ${ }^{1 *}$, Sebastian Ruder ${ }^{2,3+*}$, and Noah A. Smith ${ }^{1,4}$ \\ ${ }^{1}$ Allen Institute for Artificial Intelligence, Seattle, USA \\ ${ }^{2}$ Insight Research Centre, National University of Ireland, Galway, Ireland \\ ${ }^{3}$ Aylien Ltd., Dublin, Ireland \\ ${ }^{4}$ Paul G. Allen School of CSE, University of Washington, Seattle, USA \\ \{matthewp, noah\}@allenai.org, sebastian@ruder.io
}

\begin{abstract}
While most previous work has focused on different pretraining objectives and architectures for transfer learning, we ask how to best adapt the pretrained model to a given target task. We focus on the two most common forms of adaptation, feature extraction (where the pretrained weights are frozen), and directly finetuning the pretrained model. Our empirical results across diverse NLP tasks with two stateof-the-art models show that the relative performance of fine-tuning vs. feature extraction depends on the similarity of the pretraining and target tasks. We explore possible explanations for this finding and provide a set of adaptation guidelines for the NLP practitioner.
\end{abstract}

\section{Introduction}

Sequential inductive transfer learning (Pan and Yang, 2010; Ruder, 2019) consists of two stages: pretraining, in which the model learns a generalpurpose representation of inputs, and adaptation, in which the representation is transferred to a new task. Most previous work in NLP has focused on pretraining objectives for learning word or sentence representations (Mikolov et al., 2013; Kiros et al., 2015).

Few works, however, have focused on the adaptation phase. There are two main paradigms for adaptation: feature extraction and fine-tuning. In feature extraction (觮) the model's weights are 'frozen' and the pretrained representations are used in a downstream model similar to classic feature-based approaches (Koehn et al., 2003). Alternatively, a pretrained model's parameters can be unfrozen and fine-tuned (b) on a new task (Dai and Le, 2015). Both have benefits: enables use of task-specific model architectures and may be

\footnotetext{
${ }^{\star}$ The first two authors contributed equally.

${ }^{\dagger}$ Sebastian is now affiliated with DeepMind.
}

\begin{tabular}{lccl}
\hline \multicolumn{3}{c}{ Conditions } & \multicolumn{1}{c}{ Guidelines } \\
Pretrain & Adapt. & Task & \multicolumn{1}{c}{} \\
\hline Any & & Any & Add many task parameters \\
\hline Any & & Any & $\begin{array}{l}\text { Add minimal task parameters } \\
\text { ! Hyper-parameters }\end{array}$ \\
\hline Any & Any & Seq. / clas. & and have similar performance \\
ELMo & Any & Sent. pair & use \\
BERT & Any & Sent. pair & use \\
\hline
\end{tabular}

Table 1: This paper's guidelines for using feature extraction ( and fine-tuning (3) with ELMo and BERT. Seq.: sequence labeling. Clas.: classification. Sent. pair: sentence pair tasks.

computationally cheaper as features only need to be computed once. On the other hand, is convenient as it may allow us to adapt a general-purpose representation to many different tasks.

Gaining a better understanding of the adaptation phase is key in making the most use out of pretrained representations. To this end, we compare two state-of-the-art pretrained models, ELMo (Peters et al., 2018) and BERT (Devlin et al., 2018) using both and across seven diverse tasks including named entity recognition, natural language inference (NLI), and paraphrase detection. We seek to characterize the conditions under which one approach substantially outperforms the other, and whether it is dependent on the pretraining objective or target task. We find that and have comparable performance in most cases, except when the source and target tasks are either highly similar or highly dissimilar. We furthermore shed light on the practical challenges of adaptation and provide a set of guidelines to the NLP practitioner, as summarized in Table 1.

\section{Pretraining and Adaptation}

In this work, we focus on pretraining tasks that seek to induce universal representations suitable for any downstream task. 
Word representations Pretrained word vectors (Turian et al., 2010; Pennington et al., 2014) have been an essential component in state-of-the-art NLP systems. Word representations are often fixed and fed into a task specific model (聯), although can provide improvements (Kim, 2014). Recently, contextual word representations learned supervisedly (e.g., through MT; McCann et al., 2017) or unsupervisedly (typically through language modeling; Peters et al., 2018) have significantly improved over noncontextual vectors.

Sentence embedding methods Such methods learn sentence representations via different pretraining objectives such as previous/next sentence prediction (Kiros et al., 2015; Logeswaran and Lee, 2018), NLI (Conneau et al., 2017), or a combination of objectives (Subramanian et al., 2018). During the adaptation phase, the sentence representation is typically provided as input to a linear classifier ( ). LM pretraining with has also been successfully applied to sentence-level tasks. Howard and Ruder (2018, ULMFiT) propose techniques for fine-tuning a LM, including triangular learning rate schedules and discriminative finetuning, which uses lower learning rates for lower layers. Radford et al. (2018) extend LM- to additional sentence and sentence-pair tasks.

Masked LM and next-sentence prediction BERT (Devlin et al., 2018) combines both word and sentence representations (via masked LM and next sentence prediction objectives) in a single very large pretrained transformer (Vaswani et al., 2017). It is adapted to both word and sentence level tasks by with task-specific layers.

\section{Experimental Setup}

We compare ELMo and BERT as representatives of the two best-performing pretraining settings. This section provides an overview of our methods; see the supplement for full details.

\subsection{Target Tasks and Datasets}

We evaluate on a diverse set of target tasks: named entity recognition (NER), sentiment analysis (SA), and three sentence pair tasks, natural language inference (NLI), paraphrase detection (PD), and semantic textual similarity (STS).

NER We use the CoNLL 2003 dataset (Sang and Meulder, 2003), which provides token level an- notations of newswire across four different entity types (PER, LOC, ORG, MISC).

SA We use the binary version of the Stanford Sentiment Treebank (SST-2; Socher et al., 2013), providing sentiment labels (negative or positive) for sentences of movie reviews.

NLI We use both the broad-domain MultiNLI dataset (Williams et al., 2018) and Sentences Involving Compositional Knowledge (SICK-E; Marelli et al., 2014).

PD For paraphrase detection (i.e., decide whether two sentences are semantically equivalent), we use the Microsoft Research Paraphrase Corpus (MRPC; Dolan and Brockett, 2005).

STS We employ the Semantic Textual Similarity Benchmark (STS-B; Cer et al., 2017) and SICKR (Marelli et al., 2014). Both datasets provide a similarity value from 1 to 5 for each sentence pair.

\subsection{Adaptation}

We now describe how we adapt ELMo and BERT to these tasks. For we require a task-specific architecture, while for we need a task-specific output layer. For fair comparison, we conduct an extensive hyper-parameter search for each task.

Feature extraction (紧) For both ELMo and BERT, we extract contextual representations of the words from all layers. During adaptation, we learn a linear weighted combination of the layers (Peters et al., 2018) which is used as input to a taskspecific model. When extracting features, it is important to expose the internal layers as they typically encode the most transferable representations. For SA, we employ a bi-attentive classification network (McCann et al., 2017). For the sentence pair tasks, we use the ESIM model (Chen et al., 2017). For NER, we use a BiLSTM with a CRF layer (Lafferty et al., 2001; Lample et al., 2016).

Fine-tuning ( ELMo We max-pool over the LM states and add a softmax layer for text classification. For the sentence pair tasks, we compute cross-sentence bi-attention between the LM states (Chen et al., 2017), apply a pooling operation, then add a softmax layer. For NER, we add a CRF layer on top of the LSTM states.

Fine-tuning (b): BERT We feed the sentence representation into a softmax layer for text classification and sentence pair tasks following Devlin 


\begin{tabular}{ccrrrrrrr}
\hline \multirow{2}{*}{ Pretraining } & \multirow{2}{*}{ Adaptation } & NER & \multicolumn{2}{c}{ SA } & \multicolumn{2}{c}{ Nat. lang. inference } & \multicolumn{2}{c}{ Semantic textual similarity } \\
& & CoNLL 2003 & SST-2 & MNLI & SICK-E & SICK-R & MRPC & STS-B \\
\hline Skip-thoughts & & - & 81.8 & 62.9 & - & 86.6 & 75.8 & 71.8 \\
\hline \multirow{3}{*}{ ELMo } & & 91.7 & $\mathbf{9 1 . 8}$ & $\mathbf{7 9 . 6}$ & $\mathbf{8 6 . 3}$ & $\mathbf{8 6 . 1}$ & $\mathbf{7 6 . 0}$ & $\mathbf{7 5 . 9}$ \\
& & $\mathbf{9 1 . 9}$ & 91.2 & 76.4 & 83.3 & 83.3 & 74.7 & 75.5 \\
& $\Delta=$ & 0.2 & -0.6 & -3.2 & -3.3 & -2.8 & -1.3 & -0.4 \\
\hline \multirow{3}{*}{ BERT-base } & & 92.2 & 93.0 & $\mathbf{8 4 . 6}$ & 84.8 & 86.4 & 78.1 & 82.9 \\
& $\Delta=$ & $\mathbf{9 2 . 4}$ & $\mathbf{9 3 . 5}$ & $\mathbf{8 4 . 6}$ & $\mathbf{8 5 . 8}$ & $\mathbf{8 8 . 7}$ & $\mathbf{8 4 . 8}$ & $\mathbf{8 7 . 1}$ \\
& 0.2 & 0.5 & 0.0 & 1.0 & 2.3 & 6.7 & 4.2 \\
\hline
\end{tabular}

Table 2: Test set performance of feature extraction ( ) and fine-tuning ( compared to one sentence embedding method. Settings that are good for are colored in red $(\Delta=-1.0)$; settings good for are colored in blue $(\Delta=-1.0)$. Numbers for baseline methods are from respective papers, except for SST-2, MNLI, and STS-B results, which are from Wang et al. (2018). BERT fine-tuning results (except on SICK) are from Devlin et al. (2018). The metric varies across tasks (higher is always better): accuracy for SST-2, SICK-E, and MRPC; matched accuracy for MultiNLI; Pearson correlation for STS-B and SICK-R; and span $F_{1}$ for CoNLL 2003. For CoNLL 2003, we report the mean with five seeds; standard deviation is about $0.2 \%$.

et al. (2018). For NER, we extract the representation of the first word piece for each token and add a softmax layer.

\section{Results}

We show results in Table 2 comparing ELMo and BERT for both and approaches across the seven tasks against with Skip-thoughts (Kiros et al., 2015), which employs a next-sentence prediction objective similar to BERT.

Both ELMo and BERT outperform the sentence embedding method significantly, except on the semantic textual similarity tasks (STS) where Skipthoughts is similar to ELMo. The overall performance of and shows small differences except for a few notable cases. For ELMo, we find the largest differences for sentence pair tasks where consistently outperforms tain nearly the opposite result: significantly outperforms on all STS tasks, with much smaller differences for the others.

Discussion Past work in NLP (Mou et al., 2016) showed that similar pretraining tasks transfer better. ${ }^{1}$ In computer vision (CV), Yosinski et al. (2014) similarly found that the transferability of features decreases as the distance between the pretraining and target task increases. In this vein, Skip-thoughts-and Quick-thoughts (Logeswaran and Lee, 2018), which has similar performancewhich use a next-sentence prediction objective

\footnotetext{
${ }^{1}$ Mou et al. (2016), however, only investigate transfer between classification tasks (NLI $\rightarrow$ SICK-E/MRPC).
}

similar to BERT, perform particularly well on STS tasks, indicating a close alignment between the pretraining and target task. This strong alignment also seems to be the reason for BERT's strong relative performance on these tasks.

In $\mathrm{CV}$ generally outperforms when transferring from ImageNet supervised classification pretraining to other classification tasks (Kornblith et al., 2018). Recent results suggest is less useful for more distant target tasks such as semantic segmentation (He et al., 2018). This is in line with our results, which show strong performance with between closely aligned tasks (next-sentence prediction in BERT and STS tasks) and poor performance for more distant tasks (LM in ELMo and sentence pair tasks). Confounding factors may be the suitability of the inductive bias of the model architecture for sentence pair tasks and sotentially increased flexibility due to a larger number of parameters, which we will both analyze next.

\section{Analyses}

Modelling pairwise interactions LSTMs consider each token sequentially, while Transformers can relate each token to every other in each layer (Vaswani et al., 2017). This might facilitate with Transformers on sentence pair tasks, on which ELMo- performs comparatively poorly. We additionally compare different ways of encoding the sentence pair with ELMo and BERT. For ELMo, we compare encoding with and without cross-sentence bi-attention in Table 3. When 


\begin{tabular}{lcccc}
\hline & SICK-E & SICK-R & STS-B & MRPC \\
\hline ELMo- +bi-attn. & 83.8 & 84.0 & 80.2 & 77.0 \\
w/o bi-attn. & 70.9 & 51.8 & 38.5 & 72.3 \\
\hline
\end{tabular}

Table 3: Comparison of ELMO- cross-sentence embedding methods on dev. sets of sentence pair tasks.

\begin{tabular}{ccccc}
\hline & SICK-E & SICK-R & STS-B & MRPC \\
\hline BERT- joint enc. & $\mathbf{8 5 . 5}$ & 86.4 & $\mathbf{8 8 . 1}$ & $\mathbf{8 3 . 3}$ \\
separate encoding & 81.2 & $\mathbf{8 6 . 8}$ & 86.8 & 81.4 \\
\hline
\end{tabular}

Table 4: Comparison of BERTbedding methods on dev. sets of sentence pair tasks.

adapting the ELMo LSTM to a sentence pair task, modeling the sentence interactions by fine-tuning through the bi-attention mechanism provides the best performance. ${ }^{2}$ This provides further evidence that the LSTM has difficulty modeling the pairwise interactions during sequential processingin contrast to a Transformer LM that can be finetuned in this manner (Radford et al., 2018).

For BERT- - we compare joint encoding of the sentence pair with encoding the sentences separately in Table 4. The latter reduces performance, which shows that BERT representations encode cross-sentence relationships and are therefore particularly well-suited for sentence pair tasks.

Impact of additional parameters We evaluate whether adding parameters is useful for both adaptation settings on NER. We add a CRF layer (as used in and a BiLSTM with a CRF layer (as used in to both and show results in Table 5. We find that additional parameters are key for hurt performance with ${ }^{3}$ In addition, requires gradual unfreezing (Howard and Ruder, 2018) to match performance of feature extraction.

ELMo fine-tuning We found fine-tuning the ELMo LSTM to be initially difficult and required careful hyper-parameter tuning. Once tuned for one task, other tasks have similar hyperparameters. Our best models used slanted triangular learning rates and discriminative fine-tuning (Howard and Ruder, 2018) and in some cases gradual unfreezing.

\footnotetext{
${ }^{2}$ This is similar to text classification tasks, where we find max-pooling to outperform using the final hidden state, similar to (Howard and Ruder, 2018).

3 in fact optimizes a larger number of parameters than , so a reduced expressiveness does not explain why it underperforms on dissimilar settings.
}

\begin{tabular}{lr}
\hline Model configuration & $\mathbf{F}_{1}$ \\
\hline + BiLSTM + CRF & $\mathbf{9 5 . 5}$ \\
+ CRF & 91.9 \\
\hline + CRF + gradual unfreeze & $\mathbf{9 5 . 5}$ \\
+ BiLSTM + CRF + gradual unfreeze & 95.2 \\
+ CRF & 95.1 \\
\hline
\end{tabular}

Table 5: Comparison of CoNLL 2003 NER development set performance $\left(F_{1}\right)$ for ELMo for both feature extraction and fine-tuning. All results averaged over five random seeds.

\begin{tabular}{lccccc}
\hline & TE & GO & TR & FI & SL \\
\hline BERT- & 84.4 & 86.7 & 86.1 & 84.5 & 80.9 \\
$\Delta=-1.1$ & -0.2 & -0.6 & 0.4 & -0.6 \\
JS div & 0.21 & 0.18 & 0.14 & 0.09 & 0.09 \\
\hline
\end{tabular}

Table 6: Accuracy of feature extraction ( ference compared to fine-tuning (3) with BERT-base trained on training data of different MNLI domains and evaluated on corresponding dev sets. TE: telephone. FI: fiction. TR: travel. GO: government. SL: slate.

Impact of Target Domain Pretrained language model representations are intended to be universal. However, the target domain might still impact the adaptation performance. We calculate the Jensen-Shannon divergence based on term distributions (Ruder and Plank, 2017) between the domains used to train BERT (books and Wikipedia) and each MNLI domain. We show results in Table 6. We find no significant correlation. At least for this task, the distance of the source and target domains does not seem to have a major impact on the adaptation performance.

Representations at different layers In addition, we are interested how the information in the different layers of the models develops over the course of fine-tuning. We measure this information in two ways: a) with diagnostic classifiers (Adi et al., 2017); and b) with mutual information (MI; Noshad et al., 2018). Both methods allow us to associate the hidden activations of our model with a linguistic property. In both cases, we use the mean of the hidden activations of BERT-base ${ }^{4}$ of each token / word piece of the sequence(s) as

\footnotetext{
${ }^{4}$ We show results for BERT as they are more inspectable due to the model having more layers. Trends for ELMo are similar.
} 
the representation. ${ }^{5}$

With diagnostic classifiers, for each example, we extract the pretrained and fine-tuned representation at each layer as features. We use these features as input to train a logistic regression model (linear regression for STS-B, which has real-valued outputs) on the training data of two single sentence (CoLA ${ }^{6}$ and SST-2) and two pair sentence tasks (MRPC and STS-B). We show its performance on the corresponding dev sets in Figure 1.

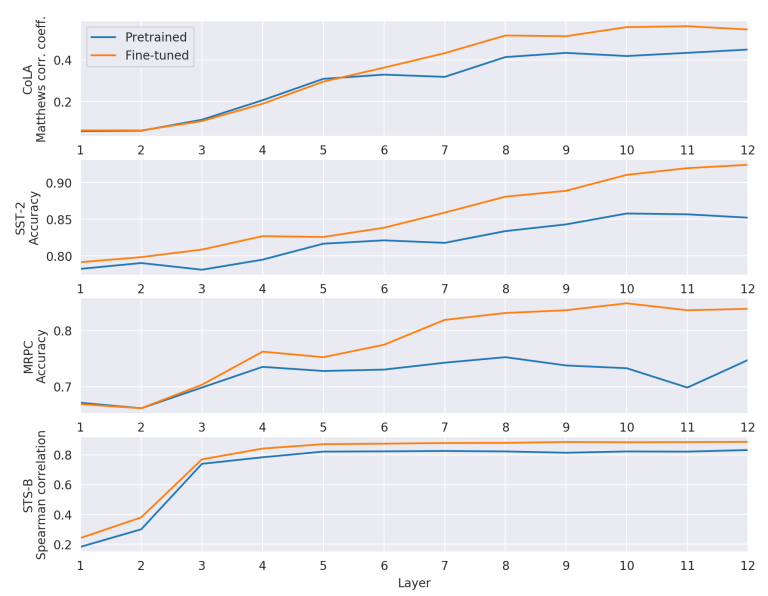

Figure 1: Performance of diagnostic classifiers trained on pretrained and fine-tuned BERT representations at different layers on the dev sets of the corresponding tasks.

For all tasks, diagnostic classifier performance generally is higher in higher layers of the model. Fine-tuning improves the performance of the diagnostic classifier at every layer. For the single sentence classification tasks CoLA and SST-2, pretrained performance increases gradually until the last layers. In contrast, for the sentence pair tasks MRPC and STS-B performance is mostly flat after the fourth layer. Relevant information for sentence pair tasks thus does not seem to be concentrated primarily in the upper layers of pretrained representations, which could explain why fine-tuning is particularly useful in these scenarios.

Computing the mutual information with regard to representations of deep neural networks has

\footnotetext{
${ }^{5}$ We observed similar results when using max-pooling or the representation of the first token.

${ }^{6}$ The Corpus of Linguistic Acceptability (CoLA; Warstadt et al., 2018) consists of examples of expert English sentence acceptability judgments drawn from 22 books and journal articles on linguistic theory. It uses the Matthews correlation coefficient (Matthews, 1975) for evaluation and is available at: nyu-mll.github.io/CoLA
}

only become feasible recently with the development of more sophisticated MI estimators. In our experiments, we use the state-of-the-art ensemble dependency graph estimator (EDGE; Noshad et al., 2018) with default hyper-parameter values. As a sanity check, we compute the MI between hidden activations and random labels and random representations and random labels, which yields 0 in every case as we would expect. ${ }^{7}$

We show the mutual information $I(H ; Y)$ between the pretrained and fine-tuned mean hidden activations $H$ at each layer of BERT and the output labels $Y$ on the dev sets of CoLA, SST-2, and MRPC in Figure 2.

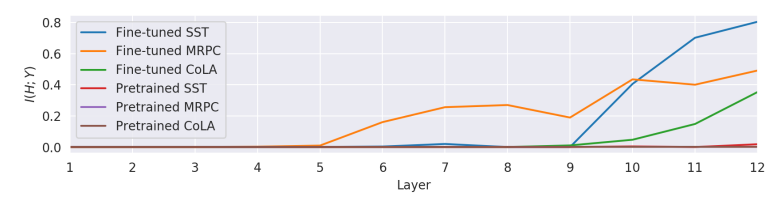

Figure 2: The mutual information between fine-tuned and pretrained mean BERT representations at different layers and the labels on the dev set of the corresponding tasks.

The MI between pretrained representations and labels is close to 0 across all tasks and layers, except for SST. In contrast, fine-tuned representations display much higher MI values. The MI for fine-tuned representations rises gradually through the intermediate and last layers for the sentence pair task MRPC, while for the single sentence classification tasks, the MI rises sharply in the last layers. Similar to our findings with diagnostic classifiers, knowledge for single sentence classification tasks thus seems mostly concentrated in the last layers, while pair sentence classification tasks gradually build up information in the intermediate and last layers of the model.

\section{Conclusion}

We have empirically analyzed fine-tuning and feature extraction approaches across diverse datasets, finding that the relative performance depends on the similarity of the pretraining and target tasks. We have explored possible explanations and provided practical recommendations for adapting pretrained representations to NLP practicioners.

\footnotetext{
${ }^{7}$ For the same settings, we obtain non-zero values with earlier estimators (Saxe et al., 2018), which seem to be less reliable for higher numbers of dimensions.
} 


\section{References}

Yossi Adi, Einat Kermany, Yonatan Belinkov, Ofer Lavi, and Yoav Goldberg. 2017. Fine-grained analysis of sentence embeddings using auxiliary prediction tasks. In Proceedings of ICLR.

Daniel M. Cer, Mona T. Diab, Eneko Agirre, Iñigo Lopez-Gazpio, and Lucia Specia. 2017. Semeval2017 task 1: Semantic textual similarity multilingual and crosslingual focused evaluation. In Proceedings of SemEval.

Qian Chen, Xiaodan Zhu, Zhenhua Ling, Si Wei, Hui Jiang, and Diana Inkpen. 2017. Enhanced LSTM for natural language inference. In Proceedings of ACL.

Alexis Conneau, Douwe Kiela, Holger Schwenk, Loïc Barrault, and Antoine Bordes. 2017. Supervised learning of universal sentence representations from natural language inference data. In Proceedings of EMNLP.

Andrew M. Dai and Quoc V. Le. 2015. Semisupervised sequence learning. In NIPS.

Jacob Devlin, Ming-Wei Chang, Kenton Lee, and Kristina Toutanova. 2018. BERT: Pre-training of deep bidirectional transformers for language understanding. In Proceedings of NAACL.

William B. Dolan and Chris Brockett. 2005. Automatically constructing a corpus of sentential paraphrases. In Proceedings of the Third International Workshop on Paraphrasing.

Matt Gardner, Joel Grus, Mark Neumann, Oyvind Tafjord, Pradeep Dasigi, Nelson F. Liu, Matthew Peters, Michael Schmitz, and Luke S. Zettlemoyer. 2017. AllenNLP: A deep semantic natural language processing platform.

Kaiming He, Ross Girshick, and Piotr Dollár. 2018. Rethinking ImageNet pre-training. arXiv preprint arXiv:1811.08883.

Jeremy Howard and Sebastian Ruder. 2018. Universal language model fine-tuning for text classification. In Proceedings of ACL.

Yoon Kim. 2014. Convolutional neural networks for sentence classification. Proceedings of EMNLP, pages 1746-1751.

Diederik P. Kingma and Jimmy Ba. 2015. Adam: A method for stochastic optimization. In Proceedings of ICLR.

Ryan Kiros, Yukun Zhu, Ruslan Salakhutdinov, Richard S. Zemel, Antonio Torralba, Raquel Urtasun, and Sanja Fidler. 2015. Skip-thought vectors. In NIPS.

Philipp Koehn, Franz Josef Och, and Daniel Marcu. 2003. Statistical phrase-based translation. In Proceedings of NAACL.
Simon Kornblith, Jonathon Shlens, Quoc V Le, and Google Brain. 2018. Do better ImageNet models transfer better? arXiv preprint arXiv:1805.08974.

John D. Lafferty, Andrew McCallum, and Fernando Pereira. 2001. Conditional random fields: Probabilistic models for segmenting and labeling sequence data. In Proceedings of ICML.

Guillaume Lample, Miguel Ballesteros, Sandeep Subramanian, Kazuya Kawakami, and Chris Dyer. 2016. Neural architectures for named entity recognition. In Proceedings of NAACL-HLT.

Lajanugen Logeswaran and Honglak Lee. 2018. An efficient framework for learning sentence representations. In Proceedings of ICLR.

Ilya Loshchilov and Frank Hutter. 2017. Fixing weight decay regularization in Adam. CoRR, abs/1711.05101.

Marco Marelli, Stefano Menini, Marco Baroni, Luisa Bentivogli, Raffaella Bernardi, Roberto Zamparelli, et al. 2014. A SICK cure for the evaluation of compositional distributional semantic models. In Proceedings of LREC.

Brian W. Matthews. 1975. Comparison of the predicted and observed secondary structure of 44 phage lysozyme. Biochimica et Biophysica Acta (BBA)Protein Structure, 405(2):442-451.

Bryan McCann, James Bradbury, Caiming Xiong, and Richard Socher. 2017. Learned in translation: Contextualized word vectors. In NIPS.

Tomas Mikolov, Ilya Sutskever, Kai Chen, Greg S Corrado, and Jeff Dean. 2013. Distributed representations of words and phrases and their compositionality. In NIPS.

Lili Mou, Zhao Meng, Rui Yan, Ge Li, Yan Xu, Lu Zhang, and Zhi Jin. 2016. How transferable are neural networks in NLP applications? Proceedings EMNLP.

Morteza Noshad, Yu Zeng, and Alfred O. Hero III. 2018. Scalable mutual information estimation using dependence graphs. arXiv preprint arXiv:1801.09125.

Sinno Jialin Pan and Qiang Yang. 2010. A survey on transfer learning. IEEE Transactions on Knowledge and Data Engineering, 22(10):1345-1359.

Jeffrey Pennington, Richard Socher, and Christopher D. Manning. 2014. GloVe: Global vectors for word representation. In Proceedings of EMNLP.

Matthew E. Peters, Mark Neumann, Mohit Iyyer, Matt Gardner, Christopher Clark, Kenton Lee, and Luke Zettlemoyer. 2018. Deep contextualized word representations. In Proceedings of NAACL-HLT. 
Alec Radford, Karthik Narasimhan, Tim Salimans, and Ilya Sutskever. 2018. Improving language understanding by generative pre-training.

Sebastian Ruder. 2019. Neural Transfer Learning for Natural Language Processing. Ph.D. thesis, National University of Ireland, Galway.

Sebastian Ruder and Barbara Plank. 2017. Learning to select data for transfer learning with Bayesian optimization. In Proceedings EMNLP.

Erik F. Tjong Kim Sang and Fien De Meulder. 2003. Introduction to the CoNLL-2003 shared task: Language-independent named entity recognition. In Proceedings of CoNLL.

Andrew M Saxe, Yamini Bansal, Joel Dapello, Madhu Advani, Artemy Kolchinsky, Brendan D Tracey, and David D Cox. 2018. On the Information Bottleneck Theory of Deep Learning. In Proceedings of ICLR 2018 .

Richard Socher, Alex Perelygin, Jean Y. Wu, Jason Chuang, Christopher D. Manning, Andrew Y. Ng, and Christopher Potts. 2013. Recursive deep models for semantic compositionality over a sentiment treebank. In Proceedings of EMNLP.

Sandeep Subramanian, Adam Trischler, Yoshua Bengio, and Christopher J Pal. 2018. Learning general purpose distributed sentence representations via large scale multi-task learning. In Proceedings of ICLR.

Joseph P. Turian, Lev-Arie Ratinov, and Yoshua Bengio. 2010. Word representations: A simple and general method for semi-supervised learning. In Proceedings of ACL.

Ashish Vaswani, Noam Shazeer, Niki Parmar, Jakob Uszkoreit, Llion Jones, Aidan N. Gomez, Lukasz Kaiser, and Illia Polosukhin. 2017. Attention is all you need. In NIPS.

Alex Wang, Amanpreet Singh, Julian Michael, Felix Hill, Omer Levy, and Samuel R. Bowman. 2018. GLUE: A multi-task benchmark and analysis platform for natural language understanding.

Alex Warstadt, Amanpreet Singh, and Samuel R Bowman. 2018. Neural network acceptability judgments. arXiv preprint arXiv:1805.12471.

Adina Williams, Nikita Nangia, and Samuel Bowman. 2018. A broad-coverage challenge corpus for sentence understanding through inference. In Proceedings of NAACL.

Jason Yosinski, Jeff Clune, Yoshua Bengio, and Hod Lipson. 2014. How transferable are features in deep neural networks? In NIPS.

\section{A Experimental Details}

For fair comparison, all experiments include extensive hyper-parameter tuning. We tuned the learning rate, dropout ratio, weight decay and number of training epochs. In addition, the finetuning experiments also examined the impact of triangular learning rate schedules, gradual unfreezing, and discriminative learning rates. Hyperparameters were tuned on the development sets and the best setting evaluated on the test sets.

All models were optimized with the Adam optimizer (Kingma and $\mathrm{Ba}, 2015$ ) with weight decay fix (Loshchilov and Hutter, 2017).

We used the publicly available pretrained ELMo $^{8}$ and BERT ${ }^{9}$ models in all experiments. For ELMo, we used the original two layer bidirectional LM. In the case of BERT, we used the BERT-base model, a 12 layer bidirectional transformer. We used the English uncased model for all tasks except for NER which used the English cased model.

\section{A.1 Feature Extraction}

To isolate the effects of fine-tuning contextual word representations, all feature based models only include one type of word representation (ELMo or BERT) and do not include any other pretrained word representations.

For all tasks, all layers of pretrained representations were weighted together with learned scalar parameters following Peters et al. (2018).

NER For the NER task, we use a two layer bidirectional LSTM in all experiments. For ELMo, the output layer is a CRF, similar to a state-of-the-art NER system (Lample et al., 2016). Feature extraction for ELMo treated each sentence independently.

In the case of BERT, the output layer is a softmax to be consistent with the fine-tuned experiments presented in Devlin et al. (2018). In addition, as in Devlin et al. (2018), we used document context to extract word piece representations. When composing multiple word pieces into a single word representation, we found it beneficial to run the biLSTM layers over all word pieces before taking the LSTM states of the first word piece in each word. We experimented with other pooling operations to combine word pieces into a

\footnotetext{
${ }^{8}$ https: / / allennlp.org/elmo

${ }^{9}$ https://github.com/google-research/ bert
} 
single word representation but they did not provide additional gains.

SA We used the implementation of the biattentive classification network in AllenNLP (Gardner et al., 2017) with default hyperparameters, except for tuning those noted above. As in the fine-tuning experiments for SST-2, we used all available annotations during training, including those of sub-trees. Evaluation on the development and test sets used full sentences.

Sentence pair tasks When extracting features from ELMo, each sentence was handled separately. For BERT, we extracted features for both sentences jointly to be consistent with the pretraining procedure. As reported in Section 5 this improved performance over extracting features for each sentence separately.

Our model is the ESIM model (Chen et al., 2017), modified as needed to support regression tasks in addition to classification. We used default hyper-parameters except for those described above.

\section{A.2 Fine-tuning}

When fine-tuning ELMo, we found it beneficial to use discriminative learning rates (Howard and Ruder, 2018) where the learning rate decreased by $0.4 \times$ in each layer (so that the learning rate for the second to last layer is $0.4 \times$ the learning rate in the top layer). In addition, for SST-2 and NER, we also found it beneficial to gradually unfreeze the weights starting with the top layer. In this setting, in each epoch one additional layer of weights is unfrozen until all weights are training. These settings were chosen by tuning development set performance.

For fine-tuning BERT, we used the default learning rate schedule (Devlin et al., 2018) that is similar to the schedule used by Howard and Ruder (2018).

SA We considered several pooling operations for composing the ELMo LSTM states into a vector for prediction including max pooling, average pooling and taking the first/last states. Max pooling performed slightly better than average pooling on the development set.

Sentence pair tasks Our bi-attentive fine-tuning mechanism is similar to the the attention mechanism in the feature based ESIM model. To apply it, we first computed the bi-attention between all words in both sentences, then applied the same "enhanced" pooling operation as in (Chen et al., 2017) before predicting with a softmax. Note that this attention mechanism and pooling operation does not add any additional parameters to the network. 